\title{
Factor structure of the Yale-Brown Obsessive-Compulsive Scale (Y-BOCS) in a large sample of patients with schizophrenia or related disorders and comorbid obsessive-compulsive symptoms
}

\author{
Lindy Boyette, Marije Swets, Carin Meijer, Luuk Wouters, G.R.O.U.P. authors *,1 \\ Academic Medical Centre University of Amsterdam, Department of Psychiatry, Amsterdam, The Netherlands
}

\section{A R T I C L E I N F O}

\section{Article history:}

Received 22 February 2010

Received in revised form 7 June 2010

Accepted 29 July 2010

Available online $\mathrm{xxxx}$

\section{Keywords:}

YBOCS

Factor analysis

Reliability

Psychosis

Obsessive-compulsive disorder

\begin{abstract}
A B S T R A C T
In the past decade there has been an increasing interest in the levels of obsessive-compulsive symptoms (OCS) found in patients with schizophrenia or related disorders. The widely acknowledged gold standard measure of the severity of OCS is the content-free version of the Yale-Brown Obsessive-Compulsive Scale (YBOCS) (Goodman et al., 1989a,b). However, factor analytic research in patients with obsessive-compulsive disorder (OCD) provided varied results. So far no study has been conducted on the factor structure of the YBOCS in patients with schizophrenia. The present study addresses this issue. We administered the Y-BOCS in a sample of 217 patients with schizophrenia or related disorders and comorbid OCS who participated in a multicentre cohort study. We used principal component analysis (PCA) to explore the underlying factor structure. A two-factor solution consistent with the originally proposed scoring structure of the Y-BOCS provided the optimal fit. We also found some support for a three-factor solution consistent with earlier findings by Kim et al. and Moritz et al. (Kim et al., 1994; Moritz et al., 2002). The produced factors showed good reliability and strong correlations with the Y-BOCS Total score. However, the resistance to compulsion item failed to demonstrate adequate correlation to the Total score, a finding consistent with earlier findings in several studies with patients with OCD.
\end{abstract}

(c) 2010 Elsevier Ireland Ltd. All rights reserved.

\section{Introduction}

Growing literature suggests higher than expected levels of obsessive-compulsive symptoms (OCS) in patients with schizophrenia. Prevalence findings vary from $7.8 \%$ to as high as $55 \%$ (overview in Byerly et al., 2005; De Haan et al., 2002, 2004, 2005; Achim et al., 2009). This wide range is primarily attributable to methodological differences, among others the instrument used to assess the severity of OCS.

The most used clinician-administered interview for assessing the severity of OCS is the content-free version of the Yale-Brown ObsessiveCompulsive Scale (Y-BOCS) (Goodman et al., 1989a,b). The scale ratings are based on five OCS aspects (duration, interference, distress, resistance and control) as reported by the patient. Obsessions and compulsions are assessed separately. The originally proposed scoring structure by

\footnotetext{
* Corresponding author. Lieuwe de Haan.

E-mail address: L.deHaan@amc.nl (L. de Haan).

Genetic Risk and Outcome of Psychosis authors: René S. Kahnª Don H. Linszen ${ }^{\mathrm{b}}$, Jim van Os ${ }^{\mathrm{c}}$, Durk Wiersma ${ }^{\mathrm{d}}$; Richard Bruggeman ${ }^{\mathrm{d}}$, Wiepke Cahn ${ }^{\mathrm{a}}$, Lieuwe de Haan ${ }^{\mathrm{b}, *}$, Lydia Krabbendam ${ }^{\mathrm{c}}$, Inez Myin-Germeys ${ }^{\mathrm{c}}$. (a) Department of Psychiatry, Rudolf Magnus Institute of Neuroscience, University Medical Center Utrecht, The Netherlands (b) Academic Medical Centre University of Amsterdam, Department of Psychiatry, Amsterdam The Netherlands (c) Maastricht University Medical Centre, South Limburg Mental Health Research and Teaching Network, Maastricht The Netherlands (d) University Medical Center Groningen, Department of Psychiatry, University of Groningen The Netherlands.
}

Goodman and colleagues consists of the summary score of the obsession severity items, the summary score of the compulsion severity items and the Y-BOCS Total score, which is the sum of all severity items.

The psychometric properties of the Y-BOCS have been elaborately investigated in samples of patients with obsessive-compulsive disorder (OCD). Internal consistency of the Total score in samples of OCD patients varied from acceptable (Woody et al., 1995) to excellent (Goodman et al., 1989a; Frost et al., 1995; Storch et al., 2005). Interrater reliability for the Y-BOCS items in samples of OCD patients has proven to be sound (Goodman et al., 1989a; Frost et al., 1995; Arrindell et al., 2002). The Y-BOCS Total score showed good convergence with most measures commonly used to assess OCS in OCD patients (Goodman et al., 1989b; Storch et al., 2005; Deacon and Abramowitz, 2005), although not with all (Kim et al., 1990, 1992; Woody et al., 1995). Regarding the discriminant validity of the Y-BOCS Total score in samples of OCD patients, a moderate correlation with anxiety and moderate to strong correlations with depression were found (Goodman et al., 1989b; McKay et al., 1995; Woody et al., 1995; Deacon and Abramowitz, 2005). This finding is likely to reflect the distress and debilitation that stem from severe obsessive-compulsive pathology (Woody et al., 1995).

Factor analytic research in samples of OCD patients provided varied results. Five different factor models have been reported. First, a onefactor model, in which all items load on a single factor (Fals-Stewart, 
1992). Second, a two-factor model representing Obsessions and Compulsions, which follows the originally proposed scoring structure (McKay et al., 1995, 1998; Arrindell et al., 2002; Storch et al., 2005). Third, a two-factor model comprised of Disturbance and Severity factors, in which the items assessing interference and distress from OCS load on a factor separate from the other severity items (Amir et al., 1997; McKay et al., 1998). Fourth, a two-factor model comprised of Severity and Resistance/Control factors, in which the items assessing resistance against and perceived control over OCS load on a separate factor (Deacon and Abramowitz, 2005). Fifth, a three-factor model comprised of Severity of Obsessions, Severity of Compulsions, and Resistance to symptoms, in which the items assessing resistance to OCS, the remaining obsession severity items and the remaining compulsion severity items form three different factors (Kim et al., 1994; Moritz et al., 2002).

There is additional evidence that the items measuring resistance and (to a lesser degree) control do not fit well with the other items. Several studies demonstrate the resistance items to have the weakest loading in a two-factor model (McKay et al., 1995, 1998; Storch et al., 2005). The resistance items have also shown poor correlation with the Y-BOCS Total score (Deacon and Abramowitz, 2005). The 'resistance to compulsion' item in particular has shown to have a decreasing effect on the consistency of the Compulsion subscale (Woody et al., 1995). Furthermore, the resistance items have shown to be less sensitive to (drug and placebo) treatment than the rest of the severity items (Kim et al., 1994). Consequently, authors have advised the deletion of the resistance to compulsion item (Woody et al., 1995), both resistance items (Kim et al., 1994) or all resistance and control items (Deacon and Abramowitz, 2005).

As far as we are aware, there has only been one report on the psychometric properties of the Y-BOCS in patients with schizophrenia or related disorders and comorbid OCS (De Haan et al., 2006). De Haan et al. found good internal consistency (Cronbach's alpha 0.76-0.79) and good interrater reliability (ICC 0.78-0.87). Findings concerning the divergent validity against depressive symptoms were inconsistent. De Haan et al. found the resistance to compulsion item to have a slightly lower item-remainder correlation with the Compulsion subscale, but there was insufficient cause to delete this item. A disadvantage of this study was its relatively small sample size (37 patients with schizophrenia and comorbid OCS). Factor analysis in such a small sample size is not feasible.

Since factor analytic research of the content-free version of the YBOCS in samples of schizophrenia patients has not yet been conducted and factor analytic research in samples of OCD patients did not unanimously support the original scoring structure, the present study was undertaken to examine the Y-BOCS factor structure in a large sample of patients with schizophrenia or related disorders. Our specific goals were as follows: 1 ) to explore the factor structure in a large sample of patients with schizophrenia or related disorders and comorbid OCS and compare it to previously reported factor models in OCD samples and 2) to examine the reliability of the produced factors.

\section{Method}

\subsection{Subjects and procedures}

Patients participating in the Genetic Risk and Outcome of Psychosis (GROUP) study were included. GROUP is a multicentre cohort study that has been designed to study vulnerability and resilience factors for variation in expression and course of nonaffective psychotic disorders.

GROUP was developed and conducted by four academic centres in the Netherlands. Each academic centre formed formalized collaborations with several mental health care institutions in its region, covering more than $75 \%$ of the mental health institutes in the Netherlands. Although GROUP participants also included healthy controls, sibling and parents, this report focuses solely on the patients with non-affective psychotic disorders. Eligible patients fulfilled the following criteria: (1) age between 18 and 50 (extremes included), (2) meeting Diagnostic and Statistical Manual of Mental Disorders, Fourth Edition (DSM-IV) criteria for a non-affective psychotic disorder (schizophrenia, schizophreniform disorder, schizoaffective disorder, delusional disor- der, or psychotic disorder NOS) (3) fluent in Dutch, and (4) able and willing to give informed consent.

To increase the interrater reliability among interviewers, all interviewers met for training workshops before the study began. The training procedure consisted of didactic sessions, observation and supervised practice. In addition, procedure manuals with verbatim scripts and web-based instructional videos were always available for all interviewers. Reliability training, regular booster sessions and two extra national training days were held to minimize experimenter drift in methods. Specific attention was given to the definition of OCS. OCS were defined as persistent, repetitive, intrusive and distressful thoughts (obsessions) not related to the patient's delusions, or repetitive goal-directed rituals (compulsions) clinically distinguishable from schizophrenic mannerisms or posturing.

\subsection{Instruments}

\subsection{1. $\mathrm{CASH} / \mathrm{SCAN}$}

Two diagnostic instruments were used to assess DSM-IV diagnosis related to psychosis. Three sites used the Comprehensive Assessment of Symptoms and History (CASH) (Andreasen et al., 1992) and one site used the Schedules for Clinical Assessment for Neuropsychiatry (SCAN 2.1) (Wing et al., 1990).

\subsubsection{Y-BOCS}

The Y-BOCS (Goodman et al., 1989a,b) is a 13-item semi-structured interview to measure the severity of OCS over the previous week. The preliminary item evaluates the occurrence of obsessions and/or compulsions, offering a comprehensive definition and examples. When OCS is present, the interview continues with five items that examine the severity of obsessions and five items that examine the severity of compulsions. These items address time spent on, interference from, distress from, resistance against and perceived control over obsessions or compulsions. Unlike some other OCS measures, the Y-BOCS considers a greater resistance to OCS as a symptom of health. All ten severity items are rated on a five-point Likert-scale, ranging from 0 (no symptom) to 4 (extreme symptoms). The interview concludes with two observational items: a clinical judgment of the severity of the disorder and a clinical judgment of the reliability of the received information.

The originally proposed scoring structure consists of three summary scores: (1) Obsession (range $=0-20$ ), which is the sum of the five items that assess the severity of obsessions, (2) Compulsion (range $=0-20$ ), which is the sum of the five items that assess the severity of compulsions and (3) the Total score (range $=0-40$ ), which is the sum of all ten severity items. A Total score of 10-20 indicates mild OCS, 21-30 indicates moderate OCS and 31-40 indicates severe OCS. The Dutch translation of the Y-BOCS (Arrindell et al., 2002) was used in this study.

\subsection{Statistical analyses}

A principal component analysis (PCA) was used to explore the factor structure of the ten Y-BOCS severity items, followed by varimax rotation. Criteria for retention of factors were eigenvalue $>1$ (Kaiser's criterion), Catell's Scree test and Parallel Analysis.

Reliability of the produced factors was assessed with Cronbach's alpha. Correlations between the produced factors with the Y-BOCS Total score were calculated. For all ten severity items we then calculated corrected item-total correlations with (a) the corresponding subscale score and (b) the Y-BOCS Total score.

\section{Results}

\subsection{Sample characteristics}

In total, 1117 patients with schizophrenia or related disorders were asked whether they had experienced obsessions or compulsions in the last week. Of this total 230 patients (20.6\%) reported OCS in the last week. The Y-BOCS was administered for this group. We excluded patients with missing data in the ten severity items and 217 patients remained. Interviewers considered reliability of the received information good to excellent for $92 \%(N=193)$ of the patients. Table 1 presents patient socio-demographic variables. Table 2 presents clinical variables related to psychosis and OCS. On average, the patients with schizophrenia or related disorders and comorbid OCS experienced mild levels of OCS (Total score M 11.7, SD 6.5).

\subsection{Exploratory factor analysis}

A principal component analysis (PCA) was used to explore the factor structure of the ten Y-BOCS severity items. The Keyser-MeyerOlkin measure of sampling adequacy revealed a score of 0.78 . Bartlett's Test of Sphericity was highly significant $\left(\chi^{2}=1065 ; \mathrm{p}<.001\right)$. Both findings indicate that the variables entered were adequate for factor 
Table 1

Patient socio-demographic variables $(\mathrm{N}=217)$

\begin{tabular}{lc}
\hline Characteristics & \\
\hline Age (M, SD) & $26.7(7.7)$ \\
Gender (\% female) & 24.0 \\
Decent (\%) & 74.2 \\
The Netherlands & 6.0 \\
Morocco & 2.8 \\
Surinam & 2.3 \\
Turkey & 0.5 \\
The Antilles & 12.0 \\
Other & 2.3 \\
Unknown & 15.6 \\
Highest education (\%) & 58.5 \\
Primary or less & 24.0 \\
Secondary & 1.8 \\
Tertiary or more & $94.2(16.9)$ \\
$\quad$ Unknown &
\end{tabular}

${ }^{a}$ Estimated with the short four-subtest version of the WAIS-III.

analysis (Kaiser, 1974) (Bartlett, 1954). PCA revealed the presence of three components with eigenvalues exceeding one; 3.47, 2.85 and 1.03. An inspection of the screeplot revealed a clear break after the second component and a lesser defined break after the third component. Parallel Analysis showed only two components with eigenvalues exceeding the corresponding criterion values for a randomly generated data matrix of the same size. PCA was then repeated, using varimax rotation. Models were computed in which both a two-factor (Table 3 ) and a three-factor (Table 4) solution were estimated. The two-factor solution revealed a distribution in obsessions and compulsions. The three-factor solution showed a third factor containing the two resistance items. Communality statistics supported both models, but was in slight favour of the three-factor solution. However, this three-factor distribution was not as consistent as the distribution in the two-factor solution. Considering this, and also taking the result of the Parallel Analysis into account, the findings of this study provide a stronger support for the use of the original scoring structure comprised of two factors: Obsession and Compulsion.

\subsection{Reliability}

Cronbachs alpha coefficient of the Y-BOCS Total score in the current sample was an acceptable 0.70 . The subscales Obsession and Compulsion produced good reliability coefficients; 0.85 and 0.81 respectively. The deletion of the resistance to obsession item resulted

Table 2

Patient clinical variables $(\mathrm{N}=217)$.

\begin{tabular}{lc}
\hline Characteristics & \\
\hline DSM diagnosis (\%) & \\
$\quad$ Schizophrenia & 71.9 \\
Schizophreniform disorder & 4.1 \\
Schizoaffective disorder & 11.1 \\
Delusional disorder & 0.5 \\
Psychotic disorder NOS & 10.1 \\
Other psychotic disorder & 2.3 \\
Status antipsychotics (\% currently using) & 81.6 \\
Duration of illness (M, SD) & $5.5(4.8)$ \\
Age of onset first psychotic episode (M, SD) & $20.6(7.3)$ \\
Number of psychotic episodes (M, SD) & $1.8(1.0)$ \\
Y-BOCS scale scores (M, SD) & \\
Obsession subscale & $5.9(5.0)$ \\
Compulsion subscale & $5.8(4.7)$ \\
Total Score & $11.7(6.5)$ \\
Total score ratings (\%) & \\
Mild (10-20) & 44.7 \\
Moderate (21-30) & 11.1 \\
Severe (31-40) & 1.0 \\
\hline
\end{tabular}

a Onset first psychotic episode until date of testing, calculated in years.
Table 3

PCA two-factor solution model of the Y-BOCS.

\begin{tabular}{lcc}
\hline Item & Factor I & Factor II \\
\hline 1. Time spent on obsession & $\mathbf{0 . 8 5}$ & -0.09 \\
2. Interference from obsession & $\mathbf{0 . 8 4}$ & -0.11 \\
3. Distress from obsession & $\mathbf{0 . 8 8}$ & -0.19 \\
4. Resistance to obsession & $\mathbf{0 . 6 1}$ & 0.11 \\
5. Control over obsession & $\mathbf{0 . 8 0}$ & 0.10 \\
6. Time spent on compulsion & -0.06 & $\mathbf{0 . 7 8}$ \\
7. Interference from compulsion & 0.17 & $\mathbf{0 . 6 9}$ \\
8. Distress from compulsion & -0.02 & $\mathbf{0 . 8 6}$ \\
9. Resistance to compulsion & -0.22 & $\mathbf{0 . 6 5}$ \\
10. Control over compulsion & -0.01 & $\mathbf{0 . 8 4}$ \\
\hline
\end{tabular}

Note: Factor loadings $\geq 0.50$ are set in bold type.

in a slight increase in the Cronbach's alpha of the Obsession subscale (0.87). The deletion of the resistance to compulsion item resulted in a slight increase in the Cronbach's alpha of the Compulsion subscale (0.82) and the Total score (0.73). The deletion of any other item resulted in a decrease in internal consistency.

There was no significant correlation between the Obsession and Compulsion subscales $(r=-0.09, P=0.17)$. Correlations between the Obsession subscale and the Compulsion subscale with the Y-BOCS Total score were strong; respectively 0.70 and $0.64(\mathrm{p}<0.01)$. With regard to item-total correlations, each item was at least moderately related to its corresponding subscale (see Table 5). However, the resistance to compulsion item failed to demonstrate adequate correlation to the Y-BOCS Total score.

\section{Discussion}

This study focuses on OCS/OCD in patients with schizophrenia and related disorders and more specifically on the factor structure of a widely used instrument for OCS severity: the Y-BOCS. Despite the general acknowledgement of the Y-BOCS as the golden standard for OCS severity, some basic psychometric questions remained unanswered. Factor analytic research in samples of OCD patients did not unanimously support the original scoring structure. Furthermore, there has been no factor analytic research conducted in a sample of patients with schizophrenia or related disorders. The present study addressed this issue in a sample of 217 patients with schizophrenia or related disorders and comorbid OCS. First we conducted exploratory factor analysis to examine the underlying factor structure of the YBOCS. We then examined the reliability of the produced factors.

Principal Component Analysis revealed a clearly defined twofactor model with both components showing a number of strong loadings and all variables loading substantially on one component. The interpretation of the two components is consistent with the originally proposed scoring structure of the Y-BOCS (Goodman et al., 1989a) and was supported by the findings of later confirmatory factor analysis in samples of OCD patients (McKay et al., 1995, 1998;

Table 4

PCA three-factor solution model of the Y-BOCS.

\begin{tabular}{lccr}
\hline Item & Factor I & Factor II & Factor III \\
\hline 1. Time spent on obsession & $\mathbf{0 . 8 7}$ & -0.01 & -0.01 \\
2. Interference from obsession & $\mathbf{0 . 8 8}$ & -0.00 & -0.09 \\
3. Distress from obsession & $\mathbf{0 . 8 9}$ & -0.13 & 0.04 \\
4. Resistance to obsession & $\mathbf{0 . 5 0}$ & -0.05 & $\mathbf{0 . 6 4}$ \\
5. Control over obsession & $\mathbf{0 . 7 2}$ & 0.01 & 0.48 \\
6. Time spent on compulsion & -0.08 & $\mathbf{0 . 8 2}$ & -0.01 \\
7. Interference from compulsion & 0.20 & $\mathbf{0 . 8 2}$ & -0.22 \\
8. Distress from compulsion & -0.09 & $\mathbf{0 . 8 3}$ & 0.22 \\
9. Resistance to compulsion & -0.35 & 0.46 & $\mathbf{0 . 5 8}$ \\
10. Control over compulsion & -0.12 & $\mathbf{0 . 7 3}$ & 0.45 \\
\hline
\end{tabular}

Note: Factor loadings $\geq 0.50$ are set in bold type. 
Table 5

Corrected item-total correlations of the Y-BOCS.

\begin{tabular}{|c|c|c|}
\hline \multirow[t]{2}{*}{ Item } & \multirow{2}{*}{$\begin{array}{l}\text { Corrected item-total } \\
\text { correlation } \\
\text { Corresponding subscale }\end{array}$} & \multirow{2}{*}{$\begin{array}{l}\text { Corrected item-total } \\
\text { correlation } \\
\text { Total score }\end{array}$} \\
\hline & & \\
\hline \multicolumn{3}{|l|}{ Obsession subscale } \\
\hline 1. Time spent on obsession & 0.72 & 0.40 \\
\hline 2. Interference from obsession & 0.70 & 0.37 \\
\hline 3. Distress from obsession & 0.77 & 0.35 \\
\hline 4. Resistance to obsession & 0.49 & 0.39 \\
\hline 5. Control over obsession & 0.70 & 0.52 \\
\hline \multicolumn{3}{|l|}{ Compulsion subscale } \\
\hline 6. Time spent on compulsion & 0.63 & 0.33 \\
\hline 7. Interference from compulsion & 0.49 & 0.40 \\
\hline 8. Distress from compulsion & 0.73 & 0.40 \\
\hline 9. Resistance to compulsion & 0.51 & 0.15 \\
\hline 10. Control over compulsion & 0.74 & 0.42 \\
\hline
\end{tabular}

Note: Correlations $<0.30$ are set in bold type.

Arrindell et al., 2002; Storch et al., 2005). We also found some support for a three-factor model found with exploratory factor analysis in samples of OCD patients (Kim et al., 1994; Moritz et al., 2002). However, this distribution was not as consistent as the distribution in the two-factor solution. Parallel Analysis also indicated a two-factor solution. Parallel Analysis has shown to be the most accurate in choosing the number of components to retain, with both Kaiser's criterion and Catell's Scree Test tending to overestimate the number of components (Zwick and Velicer, 1986; Hubbard and Allen, 1987). Therefore the original scoring structure as suggested by the Y-BOCS authors provides the optimal fit in the present sample.

The produced factors showed good reliability coefficients and strong correlations with the Y-BOCS Total score. Each Y-BOCS item was at least moderately related to its corresponding subscale. However, the resistance to compulsion item failed to demonstrate adequate correlation to the Y-BOCS Total score. This finding is consistent with prior research in samples of OCD patients (Woody et al., 1995; Deacon and Abramowitz, 2005). In the former sole study of the psychometric qualities of the Y-BOCS in a sample of patients with schizophrenia and comorbid OCS, De Haan et al. (2006) found the resistance to compulsion item to have a slightly lower itemremainder correlation with the Compulsion subscale, but there was an insufficient cause to delete this item. In the present study we find stronger support for the deletion of this item, since exclusion would bring a more consistent item-loading pattern and a more stable factor structure.

So we find indication that the resistance to compulsion item measures something different than the Y-BOCS as a whole. To make it more specific: in a significant amount of patients a greater resistance to compulsions is not a sign of less severe OCS. It might be that a subgroup exists in which less resistance reflects more severe obsessivecompulsive pathology. Some authors have speculated in this area and have suggested that the level of insight in OCS could play a key role (Storch et al., 2005; Deacon and Abramowitz, 2005). It is highly plausible that an individual is less likely to attempt to resist a compulsion if he or she believes that the execution of the compulsion is vital to prevent a catastrophe (indicating poor insight). Bellino et al. (Bellino et al., 2005) found a specific inverse correlation between insight in OCS and the severity of compulsive symptoms. Other studies found an association between lower insight and higher levels of OCS, but this was not limited to the severity of compulsions (Catapano et al., 2001; Turksoy et al., 2002). Another possible explanation might lie in the overwhelming nature of the symptoms. Woody et al. (1995) observed that some patients seemed to find it hard to differentiate between their attempts to resist OCS and their success in doing so.

Summarizing, the Y-BOCS is suitable for assessing the severity of OCS in patients with schizophrenia. This study supports the originally proposed scoring structure of the Y-BOCS in a sample of patients with schizophrenia and related disorders. Finally our findings add to the ongoing debate about the fit of the resistance items.

\section{Acknowledgement}

We are grateful for the generosity of time and effort by the patients and their families, healthy subjects, and all researchers who make this GROUP project possible. The infrastructure for the GROUP study is funded through the Geestkracht Programme of the Dutch Health Research Council (ZON-MW, grant number 10-000-1002) and matching funds from participating universities and mental health care organizations (Amsterdam: Academic Psychiatric Centre of the Academic Medical Centre and the mental health institutions: Ingeest, Arkin, Dijk en Duin, Rivierduinen, Erasmus Medical Centre, GGZ Noord Holland Noord. Maastricht: Maastricht University Medical Centre and the mental health institutions: GGZ Eindhoven, GGZ Midden-Brabant, GGZ Oost-Brabant, GGZ Noord- Midden Limburg, Mondriaan Zorggroep, Prins Clauscentrum Sittard, RIAGG Roermond, Universitair Centrum Sint-Jozef Kortenberg, CAPRI University of Antwerp, PC Ziekeren Sint-Truiden, PZ Sancta Maria Sint-Truiden, GGZ Overpelt, OPZ Rekem. Groningen: University Medical Centre Groningen and the mental health institutions: Lentis, GGZ Friesland, GGZ Drenthe, Dimence, Mediant, GGZ De Grote Rivieren and Parnassia psycho-medical centre (The Hague). Utrecht: University Medical Centre Utrecht and the mental health institutions Altrecht, Symfora, Meerkanten, Riagg Amersfoort, en Delta.)

\section{References}

Achim, A.M., Maziade, M., Raymond, E., Olivier, D., Mérette, C., Roy, M.A., 2009. How prevalent are anxiety disorders in schizophrenia? A meta-analysis and critical review on a significant association. Schizophrenia Bulletin [Electronic publication ahead of print] PubMed.

Amir, N., Foa, E.B., Coles, M.E., 1997. Factor structure of the Yale-Brown Obsessive Compulsive Scale. Psychological Assessment 9, 312-316.

Andreasen, N.C., Flaum, M., Arndt, S., 1992. The Comprehensive Assessment of Symptoms and History (CASH): an instrument for assessing diagnosis and psychopathology. Archives of General Psychiatry 49, 615-623.

Arrindell, W.A., De Vlaming, I., Eisenhardt, B.M., Van Berkum, D.E., Kwee, M.G., 2002. Cross-cultural validity of the Yale-Brown Obsessive Compulsive Scale. Journal of Behavior Therapy and Experimental Psychiatry 33, 159-176.

Bartlett, M.S., 1954. A note on the multiplying factors for various chi square approximations. Journal of the Royal Statistical Society 16, 296-298.

Bellino, S., Patria, L., Ziero, S., Bogetto, F., 2005. Clinical picture of obsessive-compulsive disorder with poor insight: a regression model. Psychiatry Research 136, 223-231.

Byerly, M., Goodman, W., Acholonu, W., Bugno, R., Rush, A.J., 2005. Obsessive compulsive symptoms in schizophrenia: frequency and clinical features. Schizophrenia Research 76, 309-316.

Catapano, F., Sperandeo, R., Perris, F., Lanzaro, M., Maj, M., 2001. Insight and resistance in patients with obsessive-compulsive disorder. Psychopathology 34, 62-68.

De Haan, L., Beuk, N., Hoogenboom, B., Dingemans, P., Linszen, D., 2002. ObsessiveCompulsive symptoms during treatment with olanzapine and risperidone, a prospective study of 113 patients with recent-onset schizophrenia or related disorders. The Journal of Clinical Psychiatry 63, 104-107.

De Haan, L., Oekeneva, A., Van Amelsvoort, T., Linszen, D., 2004. Obsessive-Compulsive Disorder and treatment with clozapine in 200 patients with recent-onset schizophrenia or related disorders. European Psychiatry 19, 524.

De Haan, L., Hoogenboom, B., Beuk, N., Dingemans, P., Van Amelsvoort, T., Linszen, D., 2005. Obsessive-compulsive symptoms distinct from positive and negative symptoms in patients with recent-onset schizophrenia. Canadian Journal of Psychiatry 50, 519-524.

De Haan, L., Hoogeboom, B., Beuk, N., Wouters, L., Dingemans, P., Linszen, D., 2006. Reliability and validity of the Yale-Brown Obsessive-Compulsive Scale in schizophrenia patients. Psychopharmacology Bulletin 39, 25-30.

Deacon, B.J., Abramowitz, J.S., 2005. The Yale-Brown Obsessive Compulsive Scale: factor analysis, construct validity, and suggestions for refinement. Journal of Anxiety Disorders 19, 573-585.

Fals-Stewart, W., 1992. A dimensional analysis of the Yale-Brown Obsessive Compulsive Scale. Psychological Reports 70, 239-240.

Frost, R.O., Steketee, G., Krause, M.S., Trepanier, K.L., 1995. The relationship of the YaleBrown Obsessive Compulsive Scale (YBOCS) to other measures of obsessive compulsive symptoms in a nonclinical population. Journal of Personality Assessment 65, 158-168.

Goodman, W.K., Price, L.H., Rasmussen, S.A., Mazure, C., Fleischmann, R.L., Hill, C.L. Heninger, G.R., Charney, D.S., 1989a. The Yale-Brown Obsessive Compulsive Scale: I. Development, use, and reliability. Archives of General Psychiatry 46, 1006-1011.

Goodman, W.K., Price, L.H., Rasmussen, S.A., Mazure, C., Delgado, P., Heninger, G.R., Charney, D.S., 1989b. The Yale-Brown Obsessive Compulsive Scale: II. Validity. Archives of General Psychiatry 46, 1012-1016.

Hubbard, R., Allen, S.J., 1987. An empirical comparison of alternative methods for principal component extraction. Journal of Business Research 15, 173-190.

Kaiser, H., 1974. An index of factorial simplicity. Psychometrika 39, 31-36.

Kim, S.W., Dysken, M.W., Kuskowski, M., 1990. The Yale-Brown Obsessive-Compulsive Scale: a reliability and validity study. Psychiatry Research 34, 99-106. 
Kim, S.W., Dysken, M.W., Kuskowski, M., 1992. The Symptom Checklist-90 ObsessiveCompulsive Subscale: a reliability and validity study. Psychiatry Research 41, $37-44$

Kim, S.W., Dysken, M.W., Pheley, A.M., Hoover, K.M., 1994. The Yale-Brown ObsessiveCompulsive Scale: measures of internal consistency. Psychiatry Research 51, 203-211.

McKay, D., Danyko, S., Neziroglu, F., Yaryura-Tobias, J.A., 1995. Factor structure of the Yale-Brown Obsessive-Compulsive Scale: a two dimensional measure. Behavior Research and Therapy 33, 865-869.

McKay, D., Neziroglu, F., Stevens, K., Yaryura-Tobias, J.A., 1998. The Yale-Brown Obsessive Compulsive Scale: confirmatory factor analytic findings. Journal of Psychopathology and Behavioural Assessment 20, 265-274.

Moritz, S., Meier, B., Kloss, M., Jacobsen, D., Wein, C., Fricke, S., Hand, I., 2002. Dimensional structure of the Yale-Brown Obsessive-Compulsive Scale (Y-BOCS). Psychiatry Research 109, 193-199.
Storch, E.A., Shapira, N.A., Dimoulas, E., Geffken, G.R., Murphy, T.K., Goodman, W.K., 2005. Yale-Brown Obsessive Compulsive Scale: the dimensional structure revisited. Depression and Anxiety 22, 28-35.

Turksoy, N., Tukel, R., Ozdemir, O., Karali, A., 2002. Comparison of clinical characteristics in good and poor insight obsessive-compulsive disorder. Journal of Anxiety Disorders 16, 413-423.

Wing, J.K., Babor, T., Brugha, T., Burke, J., Cooper, J.E., Giel, R., Jablenski, A., Regier, D., Sartorius, N., 1990. SCAN. Schedules for Clinical Assessment in Neuropsychiatry. Archives of General Psychiatry 47, 589-593.

Woody, S.R., Steketee, G., Chambless, D.L., 1995. Reliability and validity of the YaleBrown Obsessive-Compulsive Scale. Behaviour Research and Therapy 33, 597-605.

Zwick, W.R., Velicer, W.F., 1986. Comparison of five rules for determining the number of components to retain. Psychological Bulletin 99, 432-442. 